\title{
Variación en el patrón de coloración de Anablepsoides hartii (Cyprinodontiformes: Cynolebiidae) en arroyos costeros de Venezuela
}

\author{
Edwin Infante-Rivero \\ Instituto de Zoología y Ecología Tropical, Centro Museo de Biología de la Universidad Central de Venezuela, \\ Laboratorio de Ictiología, Facultad de Ciencias UCV; edwinfante@gmail.com
}

Recibido 08-VI-2017. Corregido 25-X-2017. Aceptado 20-XI-2017.

\begin{abstract}
Variation in the coloration pattern of Anablepsoides hartii (Cyprinodontiformes: Cynolebiidae) in coastal streams of Venezuela. Anablepsoides hartii is a non-annual fish of the family Cynolebiidae. Historically, this species has presented some inconsistencies for proper identification, due to a variation in the color pattern. The aim of this study was to describe and illustrate the different types of color patterns found in this species. We examined a total of 336 preserved specimens (19 lots) deposited in the ichthyological collections of the Museo de Biologia de la Universidad Central de Venezuela and the Museo de Ciencias Naturales in Guanare, Venezuela. The specimens were sorted by distinctive characteristics of size, sex and locality. Besides, we took photographs of each individual, vectorized them with detail, separating light and dark colorations and delimited color patterns of the same tone with the program Corel Draw version x7 (Spanish). My analysis showed that A. hartii presented four colors patterns. The color morphs were classified as: 1 male, 2 female, 3 intermediate and 4 montane. The first two color morphs were separated by sexual characters (sexual dimorphism); in turn, the intermediate color morph presented characters of both males and females in the same individual. Finally, the montane color morph differed from the previous ones by presenting a series of incomplete lateral rows, followed by a half-body reticulation to the tail. This analysis allowed the identification of the differences in this morphologically variable species. Rev. Biol. Trop. 66(1): 293-302. Epub 2018 March 01.
\end{abstract}

Key words: cromotype; non-annual fish; coloration; patterns.

Anablepsoides hartii (Cyprinodontiformes: Cynolebiidae; Eschmeyer, Fricke, \& Van Der Laan, 2017), fue descrito por Boulenger en 1890 con ejemplares procedentes de la Isla de Trinidad (Costa, 2003). Esta especie presenta una amplia distribución que abarca la porción sur de las Antillas y el norte de América del Sur: incluyendo Aruba, Curazao, Trinidad y Tobago, Isla de Margarita y Paria, cuencas de ríos costeros en el Caribe venezolano, así como del río Orinoco (Froese \& Pauly, 2014; Huber, 1996; Kenny, 1995; Robins et al., 1991; Costa, 2003; Lasso et al., 2004).

Históricamente, Boulenger (1890) describe esta especie con el nombre de Haplochilus hartii. Luego, Schultz (1949) describe a Rivulus bondi, quien posteriormente fue considerado sinónimo de A. hartii (Huber, 1996; Costa,
2003). Mago (1970) incluye esta especie dentro de la lista de peces de agua dulce de Venezuela. Parenti (1981) ilustra por primera vez parte de su osteología y la incluye en su trabajo titulado "Filogenia y biogeografía de los peces Cyprinodontiformes". Taphorn, Royero, MachadoAllison y Mago-Leccia (1997), actualizan la lista de peces de Venezuela e incluyen esta especie como Rivulus hartii. Más tarde, Costa (2011) restringe el género Rivulus a Cuba y ubica las especies descritas en este género en cinco nuevos géneros (Atlantirivulus, Cynodonichthys, Laimosemion, Melanorivulus y Anablepsoides) incluyendo a $R$. hartii en el género Anablepsoides.

Boulenger (1890) describe el patrón de coloración de $A$. hartii de la siguiente forma: "Brown or bronzy above, yellowish inferiorly; 
each scale with a darker spot, best defined in the males; dorsal and anal fins whitish, with grey dots, anal with a fine blackish edge; caudal grey or blackish." Tal y como es descrito, este patrón de coloración concuerda con lo observado en otras especies dentro del género. Como consecuencia, esto ha generado varias interrogantes al comparar esta especie con especies relacionadas, lo que ha ocasionado asignaciones y consideraciones erróneas en ciertos casos (e.g. la sinonimia de " $R$. bondi", Schultz, 1949; sugerida por Huber, 1996; Costa, 2003; Vermeulen, 2015).

Esta semejanza morfológica es tan marcada que en ocasiones, diferentes autores al examinar caracteres externos, han considerado la sinonimia de $A$. hartii con $A$. deltaphilus, $A$. holminae, y A. igneus (e.g. Collier, Espinoza, \& Murphy, 1998; Lasso \& Lasso-Alcalá, 2011).

En los últimos años, con el avance tecnológico, se ha incrementado el estudio morfológico de los organismos basados en el procesamiento y análisis de imágenes. Diferentes autores llaman a esta metodología procesamiento óptico digital, que tiene un alto impacto en los análisis y caracterización de diferentes representantes de varios grupos naturales (Herrera, Daza, Moreno, \& Mavilio, 2008; Herrera \& Moreno, 2014). El método ha sido usado en la microscopía y en poblaciones naturales no tan pequeñas, y se ha podido identificar individuos y estimar poblaciones con la "foto-identificación" (Seydack, 1984; Karanth, 1995; Mesa-Zavala, Álvarez-Cárdenas, Galina-Tessaro, TroyoDiéguez, \& Guerrero-Cárdenas, 2012). Por lo anteriormente expuesto, y al conocer que la variación fenotípica y morfológica en $A$. hartii no se ha revisado en suficiente detalle, presento a continuación una descripción de los diversos patrones de coloración, en preservativo, de las poblaciones de esta especie en las costas venezolanas, con el propósito de facilitar y promover futuros estudios comparativos y descriptivos dentro del grupo y áreas de estudio.

\section{MATERIALES Y MÉTODOS}

El estudio comenzó en julio del 2015, las muestras fueron separadas, fotografiadas y analizadas entre agosto y septiembre del mismo año en el laboratorio de Ictiología de la Universidad Central de Venezuela.

Para describir los patrones de coloración de $A$. hartii, se revisaron y fotografiaron un total de 336 ejemplares de 19 lotes fijados en formalina al $10 \%$ y preservados en etanol al $70 \%$. El material se encuentra depositado y catalogado en las Colecciones de Peces del Museo de Biología de la Universidad Central de Venezuela (MBUCV) y del Museo de Ciencias Naturales de Guanare (MCNG), colectadas desde 1938 al 2015, datos adicionales se presenta en el anexo al final del manuscrito. Este material proviene de diferentes localidades (estados: Sucre 3, Falcón 1, Miranda 3, Yaracuy 1, Lara 1, Nueva Esparta 2, una población extinta del Distrito Federal y una lote de la Isla de Trinidad). Adicionalmente, se incluyen en el análisis fotografías de trabajos publicados: $(e, g$. Phillip et al., 2013) y páginas electrónicas (ver Rodríguez-Olarte \& Taphorn, 2007).

La identificación de los especímenes se realizó con la comparación de las descripciones originales de Boulenger (1890), y variaciones de Schultz (1949, en la descripción de A. bondi), así como caracteres diagnósticos de fichas elaboradas por Rodríguez-Olarte y Taphorn (2007), Lasso y Lasso-Alcalá (2011) y Phillip et al. (2013).

Los especímenes examinados fueron separados por características distintivas de tamaño, sexo y localidad. Posteriormente, a cada individuo se le tomó una fotografía de ambos lados del cuerpo, con una cámara digital Kodak (Easy Share Z1012 IS de 10.1 MP). Cada fotografía seleccionada fue editada con el programa Corel Draw versión x 7 en español, donde se convirtieron en mapas de 8 bits a 300 ppp a escala de grises. Cada imagen se vectorizó 
en alta calidad a un máximo de detalle. Esta aplicación separa claros de oscuros y delimita las manchas o patrones de grises con un mismo tono, definiendo el patrón observado en líneas.

\section{RESULTADOS}

Del total de ejemplares analizados, en general se observaron cuatro tipos de variación cromática: los dos primeros separaron al macho de la hembra (diferenciación sexual), el tercero presentó atributos intersexuales, y el último, estuvo bien diferenciado de los tres anteriores. Los patrones se describen a continuación:

Cromotipo masculino: Caracterizado por presentar de 5 a 7 hileras de puntos en los costados, que pueden definir líneas completas e interrumpidas y aleta dorsal con 4 a 8 manchas irregulares, perpendiculares a los radios, visibles además en especímenes juveniles. Coloración en alcohol al 70 \%: Dorso marrón oscuro bronceado hasta un blanco lavado en el vientre. En juveniles la coloración dorsal es menos marcada que en adultos, desde marrón oscuro reticulado en el dorso, a blanco inmaculado en el vientre. Aleta dorsal pardo-grisácea, manchas oscuras perpendiculares a los radios con el borde superior blanco, visibles en juveniles. Aleta caudal oscura con bordes superior e inferior blanquecinos. Aleta anal manchada con mayor intensidad en la base, puede formar puntos o líneas irregulares (Fig. 1).

Cromotipo femenino: Caracterizado por ser más uniforme y claro que el cromotipo masculino. Presenta 7 hileras de puntos laterales bien definidos. En algunos especímenes las hileras de puntos son más claras e interrumpidas en medio del cuerpo. Aleta dorsal barreteada con 3 a 5 líneas o manchas irregulares, perpendiculares a los radios. Aleta caudal manchada de 5 a 6 semicírculos. Borde de la aleta anal conspicuo y ocelo supra-penduncular presente. Coloración en alcohol al $70 \%$ : dorso, parte superior de la cabeza y opérculo, más oscuros que el resto de cuerpo. Cuerpo de color marrón claro con puntos de marrón oscuro bien delimitado hasta el medio cuerpo, bases de los flancos y vientre rosa pálido a blanco inmaculado. Aleta dorsal manchada de marrón oscuro. Aleta caudal manchada con semicírculos o bandas verticales de marrón oscuro. Anal punteada de marrón oscuro en la base y con el borde bien delimitado de negro a gris oscuro. Ocelo suprapenduncular negro bien definido, bordeado de blanco (Fig. 2). Estas características son semejantes a los juveniles y ejemplares menores de $25 \mathrm{~mm}$, sólo difieren en presentar puntos laterales espaciados que no conforman líneas.

Cromotipo intermedio: Presenta una combinación de caracteres entre los cromotipos ya descritos (masculinos y femeninos). Dorso, parte superior de la cabeza y opérculo más oscuros que el resto del cuerpo, con 5 a 6 hileras de puntos laterales interrumpidas. Caudal oscura con bandas marginales blanquecinas con reducción del ocelo supra-peduncular. Borde de la aleta anal bien delimitado. En juveniles, el cambio se ve gradual hacia el cromotipo adulto; a menor talla $(<25 \mathrm{~mm})$ las diferencias son mínimas. Sin embargo, ya por encima de los $30 \mathrm{~mm}$ comienza a aparecer

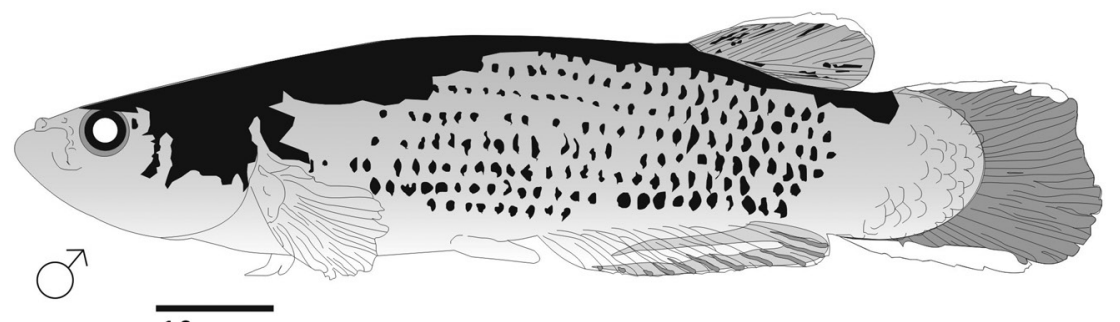

$10 \mathrm{~mm}$

Fig. 1. Cromotipo masculino de A. hartii. MBUCV V-29496, Nueva Esparta. Fig. 1. Male Chromotype of A. hartii. MBUCV V-29496, Nueva Esparta. 


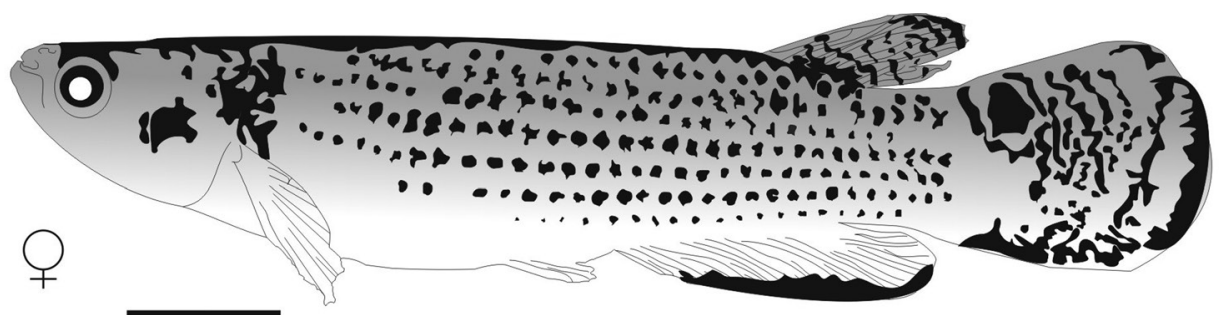

$10 \mathrm{~mm}$

Fig. 2. Cromotipo femenino de A. hartii. MBUCV V-12510, estado Sucre.

Fig. 2. Chromotype female of $A$. hartii. MBUCV V-12510, Sucre state.

la banda blanquecina por encima y por debajo de la aleta caudal, carácter del cromotipo masculino. Coloración en alcohol al 70 \%: Dorso y opérculo marrón oscuro bronceado, vientre rosa pálido. En juveniles, la coloración dorsal es menos marcada, pero mantiene el patrón. Cuerpo marrón con hilera de puntos marrón bronceado oscuro interrumpida. Aleta dorsal, con machas de marrón oscuro, perpendicular a los radios con o sin el borde superior blanco, visibles en juveniles. Aleta caudal oscura con bordes superior e inferior blanquecinos. Aleta anal manchada en la base con el borde negro (Fig. 3).

Cromotipo montano: Habitante en ecosistema riberino de alta montaña. Caracterizado por presentar de 5 a 6 hileras de puntos laterales incompletas, en la mitad anterior del cuerpo. En la mitad posterior del cuerpo, las hileras de puntos son poco evidentes, difuminadas o ausentes, y llegan a formar una completa reticulación que va desde la base de la aleta anal hasta el final del pedúnculo, más evidente en machos. Este patrón de coloración está presente en ejemplares del Distrito Capital,
Miranda y Yaracuy (MBUCV V- 4008, 19553, $3746,12517,27788,35743,35741$ y 35739 ). Coloración en alcohol al 70 \%: Marrón oscuro bronceado en cabeza, dorso y opérculo, cuerpo marrón claro con reticulaciones o ligeras bandas transversal más oscuras desde el medio cuerpo hasta la cola. Vientre rosa pálido o blanco amarillento. Hileras de puntos laterales bien definidas hasta medio cuerpo. Caudal negra con bandas superior e inferior blancas. Anal grisácea. Hembras similares al cromotipo femenino (Fig. 4).

Variación cromática: En las Figs. 5 y 6 se ilustra la variación cromática presente en individuos de diferentes localidades de las costas venezolanas; se incluye un macho de la Isla de Trinidad, algunos ejemplares de Sucre (MBUCV V- 12445, 8887, 12510) Miranda (MBUCV V- 5005), y Yaracuy (MBUCV $\mathrm{V}$ - 35738). Esta variación es marcada en la tonalidad del dorso, pero lo es menos tanto en machos, hembras y juveniles. Sobresale la coloración de las escamas cefálicas y del opérculo. Coloración en alcohol al 70 \%: Dorso marrón claro reticulado de marrón oscuro y manchado

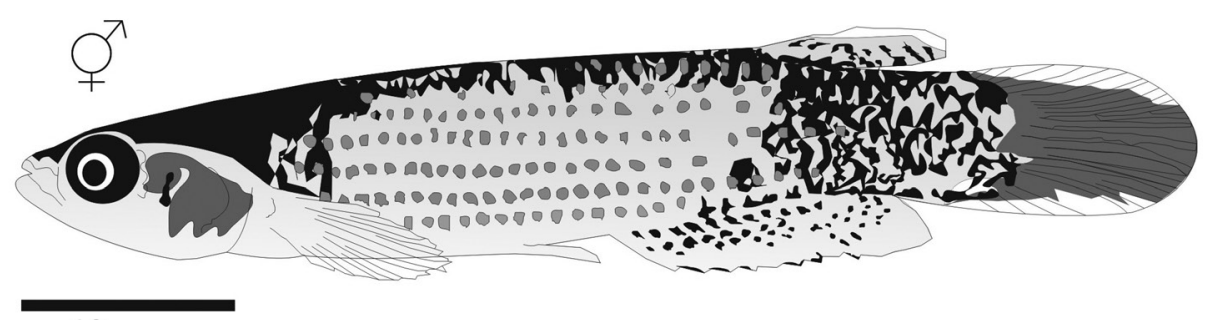

$10 \mathrm{~mm}$

Fig. 3. Cromotipo intermedio de A. hartii. Yaracuy MBUCV-V 35739 Fig. 3. Chromotype intermediate of A. hartii. MBUCV-V 35739 Yaracuy state. 

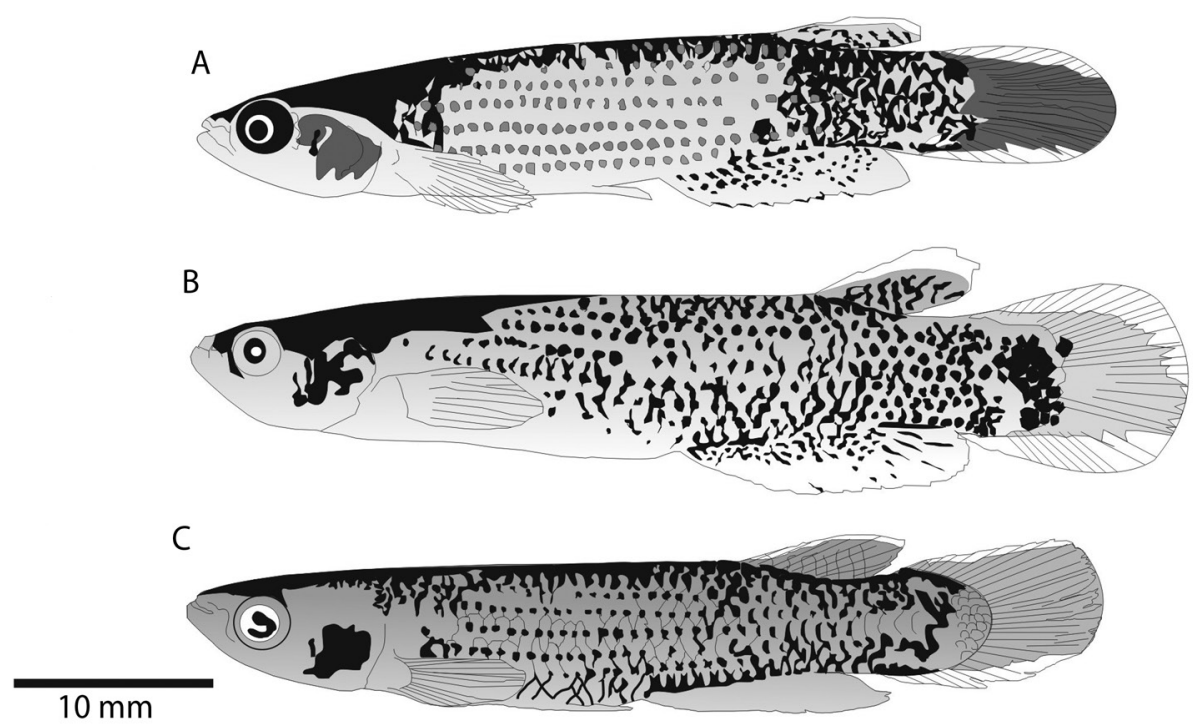

Fig. 4. Cromotipo montano. A: cromotipo montano indeterminado MBUCV -V 35739, Yaracuy. B y C: variación cromática. B: Cromotipo montano macho, MBUCV -V 35742, río Araira, Miranda. C: Cromotipo montano macho, MBUCV -V 5005, río Aricagua, Miranda.

Fig. 4. Montano chromotype. A: indeterminate montane chromotype MBUCV-V 35739, Yaracuy. B and C: color variation. B: Chromotype montane male, MBUCV-V 35742, Araira River, Miranda. C: Chromotype montano male, MBUCV-V 5005, Aricagua River, Miranda.

de blanco. Cuerpo blanquecino con 5 o 6 hileras de puntos, donde la línea de puntos más dorsal es parte del reticulado dorsal.

En ejemplares de Nueva Esparta (MBUCV V-29496) y Falcón: (MBUCV V-24109), por encima de $60 \mathrm{~mm}$ de longitud total (LT), el dorso es oscuro manchado de negro o gris; la dorsal presenta el borde blanquecino y el vientre es marrón amarillento, más intenso debajo de la cabeza, los puntos laterales son claros y se distinguen 7 hileras continuas; las aletas pectorales son de un gris amarillento con delineados entre los radios. De manera semejante, las hembras de mayor talla presentan el dorso igual o de menor intensidad que los machos. En ejemplares con el dorso más claro, la cabeza se mantiene más oscura que el resto del dorso.

A su vez, en ejemplares de tallas menores $(<50 \mathrm{~mm})$ de todas las localidades estudiadas, el dorso es marrón oscuro, es más intenso en los costados y en las escamas de la cabeza, mantiene además el patrón de coloración similar al de los adultos, pero con una menor intensidad; presenta además 4 o 5 hileras de puntos laterales. Las hembras a su vez, son mucho más claras que los machos; el dorso presenta 3 líneas bien definidas dos laterales y una central, esta última partiendo de la escamación cefálica y en los costados. Lateralmente, se distinguen 5 hileras de puntos, con el borde inferior de la aleta anal oscura, y el ocelo suprapeduncular bien definido (Fig. $6 \mathrm{c} \mathrm{y} \mathrm{d}$ ).

\section{DISCUSIÓN}

Dentro de las características distintivas de $A$. hartii, se mencionan la presencia de 6 a 8 líneas de puntos laterales que van desde el borde opercular hasta la base de la cola (Nirchio et al., 2005), y observé variación en este carácter. En ejemplares menores de $30 \mathrm{~mm}$ observé de 3 a 4 líneas interrumpidas, sólo una línea de puntos a medio cuerpo bien delimitada, usualmente más claras en los machos. En ejemplares de talla mayor a $35 \mathrm{~mm}$ de longitud total, se evidenciaron 7 hileras de puntos laterales. 


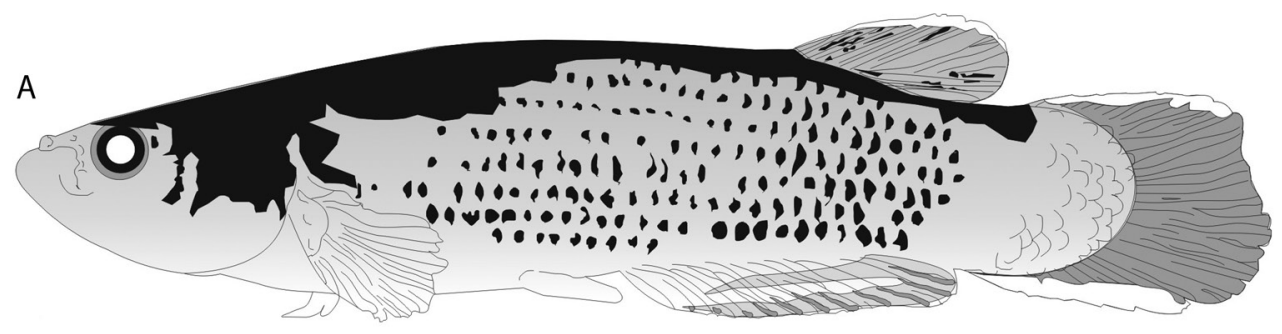

B

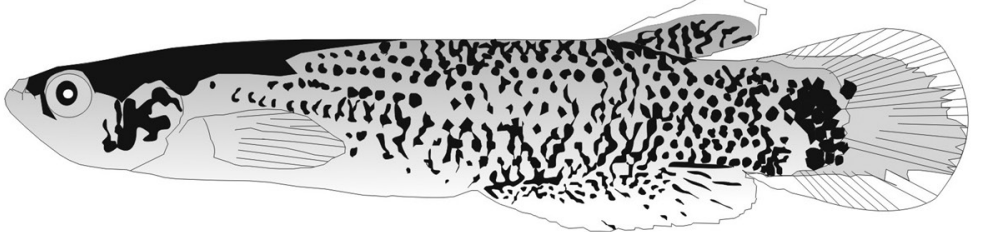

C

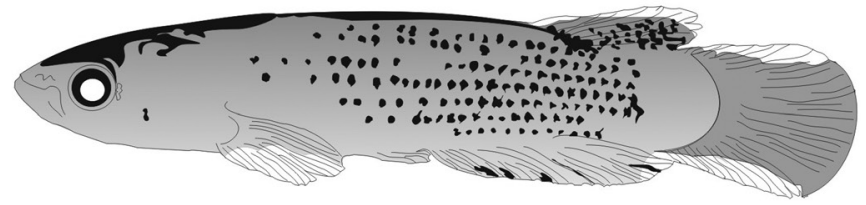

D
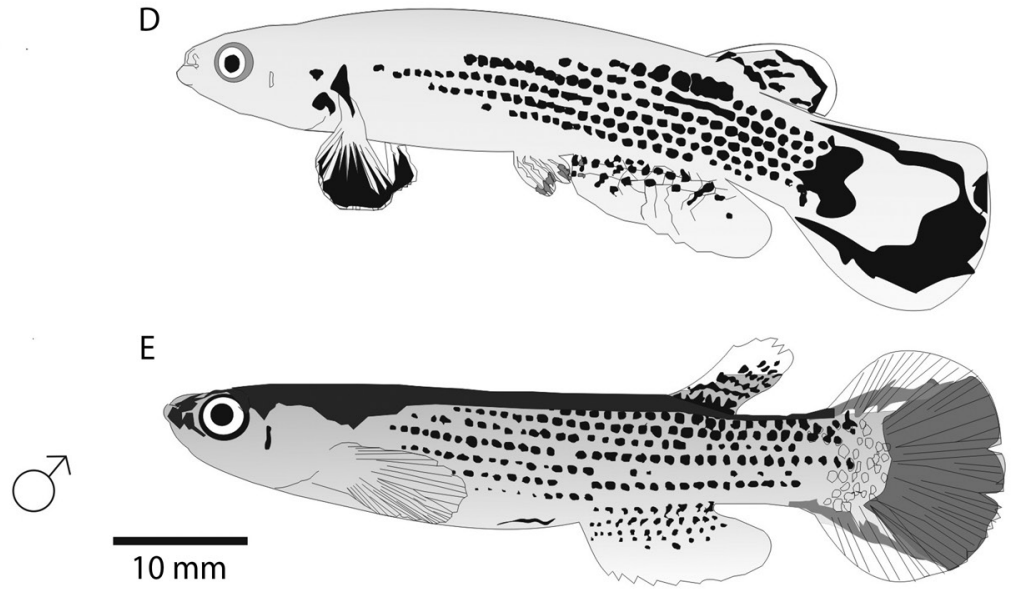

Fig. 5. Variación en cromotipo masculino A: MBUCV -V 29496, Sucre. B: MBUCV -V 35743, Miranda. C: MBUCV -V 12510 Sucre, D: tomado de: Rodríguez-Olarte y Taphorn (2007) y E: MCNG 8238 Isla de Trinidad.

Fig. 5. Variation in male chromotype A: MBUCV-V 29496, Sucre. B: MBUCV-V 35743, Miranda. C: MBUCV -V 12510 Sucre, D: taken from: Rodríguez-Olarte and Taphorn (2007) and E: MCNG 8238 Island of Trinidad.

Diferentes autores hacen mención sobre el dimorfismo sexual en cuanto al patrón de coloración (e.g. Rodríguez-Olarte \& Taphorn, 2007; Lasso \& Lasso-Alcalá, 2011; y Phillip et al., 2013), Lo que no se ha referido en la literatura, es que existe un cromotipo intermedio con caracteres tanto de machos como de hembras, presente al menos en un $18 \%$ de los juveniles, adultos y el cromotipo montano, distintivo del material examinado, y que habita en pie de monte de la vertiente del Caribe.

El cromotipo intermedio estuvo presente en casi todos los lotes analizados provenientes de las poblaciones en los estados Sucre, 
A

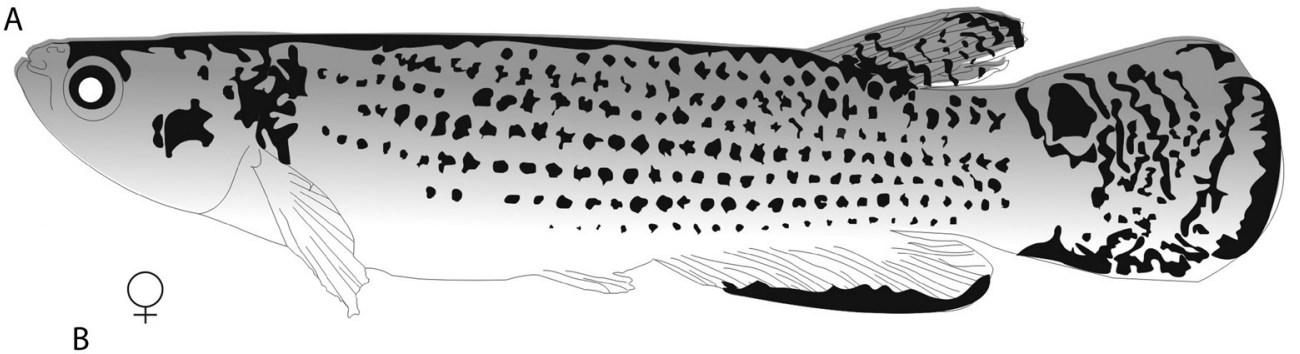

B

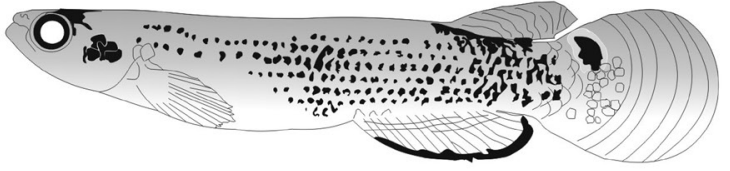

C

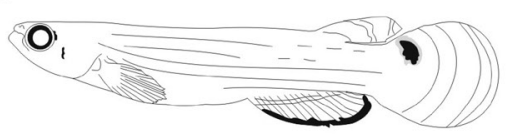

$\mathrm{E}$

D
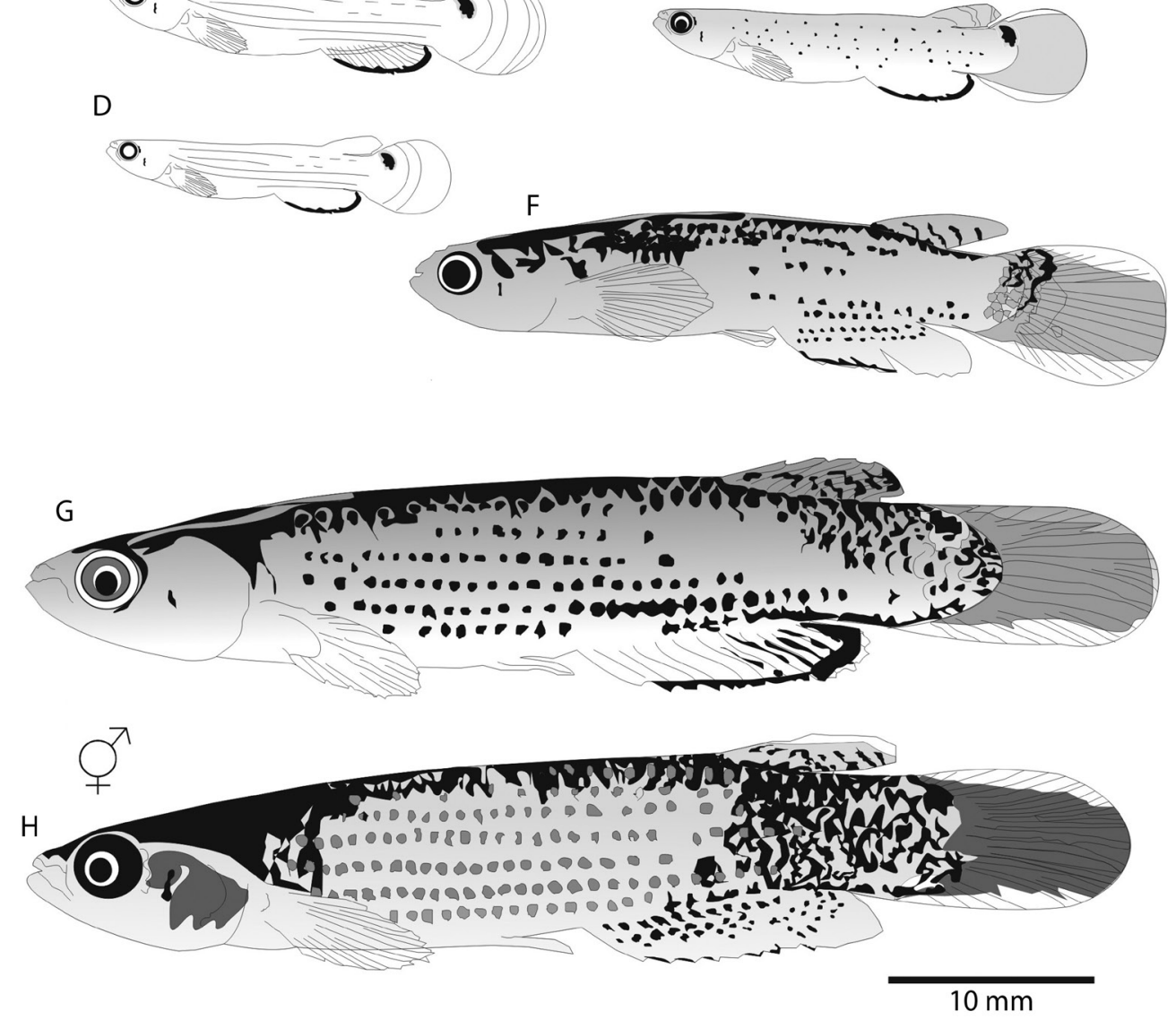

Fig. 6. Variación en cromotipo femenino (A-D), e intermedio (E-H) A: MBUCV -V 12510, Sucre B: MBUCV -V 35741, Miranda. C: MBUCV -V 24109, Falcón. D: MBUCV -V 8886, Sucre. E: MBUCV -V 20257, sucre. F: MBUCV -V 7001, Distrito Capital. G: y H: MBUCV -V 35739, Yaracuy.

Fig. 6. Variation in female chromotype (A-D), and intermediate (E-H) A: MBUCV-V 12510, Sucre B: MBUCV-V 35741, Miranda. C: MBUCV-V 24109, Falcón. D: MBUCV-V 8886, Sucre. E: MBUCV-V 20257, Sucre. F: MBUCV -V 7001, Distrito Capital. G: and H: MBUCV-V 35739, Yaracuy. 
Yaracuy, Miranda y Distrito Capital, Falcón y Nueva Esparta. Ahora bien, la relación del grupo intermedio o frecuencia de aparición del cromotipo en las poblaciones estudiadas fluctuó entre $7 \%$ y el $40 \%$ en diferentes tallas, con o sin presencia de machos. Es de relevancia recalcar que de los paratipos de Rivulus bondi (MBUCV V- 7001) colectados en el río Guaire, el $40 \%$ presentó el cromotipo intermedio con una talla menor a $35 \mathrm{~mm}$. Este cromotipo, además está presente en Sucre en los ríos: Mapuey $20 \%$, Guairiquen $24 \%$, San Juan $21 \%$, Maipire $17 \%$, Mereyes $25 \%$. En las costas de Falcón $8.5 \%$. En Yaracuy $33 \%$, al igual que en la Isla de Trinidad $33 \%$.

El hecho de encontrar un cromotipo con caracteres tanto masculinos como femeninos incita a especular que estemos frente a un representante del grupo hermafrodita secuencial protándrico, condición relativamente bien documentada en otros miembros de la familia (Nelson, Grande, \& Wilson, 2016; Guerrero-Estévez \& Moreno-Mendoza, 2010); sin embargo, en este trabajo no se presentan pruebas definitorias para verificar esta afirmación. Lo que propone a futuro es comprobar el posible cambio de sexos, así como analizar la variación morfológica y los cambios que puedan ocurrir en diferentes localidades en las que habite Anablepsoides hartii.

\section{AGRADECIMIENTOS}

El autor agradece al personal del Museo de Biología de la Universidad Central de Venezuela, así como al personal del Museo de Ciencias Naturales de Guanare Oscar Mata por permitir la revisión del material. Antonio Machado y Héctor López por sus comentarios y revisión previa del manuscrito.

\section{RESUMEN}

Anablepsoides hartii es un pez no anual de la familia Cynolebiidae. Históricamente, incongruencias en la identificación de dicha especie han surgido debido a su variación en el patrón de coloración. Por tal motivo, se describe e ilustra los diferentes tipos de patrones de coloración de esta especie. Se revisaron 336 ejemplares de 19 lotes preservados en alcohol al $70 \%$, depositado en las colecciones ictiológicas del Museo de Biología de la Universidad Central de Venezuela y el Museo de Ciencias Naturales en Guanare. Los ejemplares fueron separados por características distintivas de tamaño, sexo y localidad. Adicionalmente, se tomaron fotografías de cada individuo que fueron vectorizadas a un máximo nivel de detalle, separando claros y oscuros que delimitan los patrones con un mismo tono de coloración con el programa Corel Draw versión x 7 en español. Anablepsoides hartii presenta cuatro (4) patrones de coloración. Los cromotipos son: 1.- masculino, 2.- femenino, 3.- intermedio y 4.- montano. Los dos primeros cromotipos son separados por caracteres sexuales, a su vez el cromotipo intermedio presenta caracteres tanto de machos como de hembras en un mismo individuo. Finalmente, el cromotipo montano se diferencia del resto por presentar la serie de hileras laterales incompleta seguido por un reticulado de medio cuerpo hasta la cola. Esta contribución permite aclarar las diferencias que existen en una especie morfológicamente variable.

Palabras clave: cromotipo; pez no anual; coloración; patrones.

\section{REFERENCIAS}

Boulenger, G. A. (1890). Description of two new cyprinodontoid fishes. Annals and Magazine of Natural History London, 6, 169-170.

Collier, G. E., Espinoza, M., \& Murphy, W. J. (1998). Forensic Phylogenetics of Rivulus. Journal of the American Killifish Association, 31(2), 35-41.

Costa, W. J. E. M. (2003). Rivulidae (South American Annual Fishes). In R. E. Reis, S.O. Kullander and C.J. Ferraris, Jr. (Eds.) Checklist of the Freshwater Fishes of South and Central America (pp. 526-548.) Porto Alegre, Brasil: EDIPUCRS.

Costa, W. J. E. M. (2011). Phylogenetic position and status of Anablepsoides, Atlantirivulus, Cynodonichthys, Laimosemion and Melanorivulus (Cyprinodontiformes: Rivulidae). Icythyological Exploration of Freshwaters, 22, 233-249.

Eschmeyer, W. N., Fricke, R., \& Van Der Laan, R. (Eds.). (2017). Catalog of Fishes: Genera, Species, References. San Francisco, CA. USA: California Academy of Sciences. Retrieved from http://researcharchive. calacademy.org/research/ichthyology/catalog/fishcatmain.asp

Froese, R., \& Pauly, D. (Eds.). (2014). FishBase. World Wide Web electronic publication. Retrieved from www.fishbase.org

Guerrero-Estévez, S., \& Moreno-Mendoza, N. (2010). Sexual determination and differentiation in teleost 
fish. Reviews in Fish Biology and Fisheries, 20, 101-121.

Herrera, M., Daza, J., Moreno A., \& Mavilio, A. (Diciembre, 2008). Caracterización de muestras de materiales biológicos mediante imágenes obtenidas por técnicas interferométricas y de microscopía. La Habana Cuba: XIV Convención Científica de Ingeniería y Arquitectura.

Herrera, M., \& Moreno, A. (2014). Análisis morfológico en el procesamiento óptico-digital de imágenes para el diagnóstico de la disquinesia ciliar. Información Tecnológica, 25(1), 33-40.

Huber, J. H. (1996). Updated checklist of taxonomic names, collecting localities and bibliographic references of oviparous Cyprinodont fishes (Atherinomorpha, Pisces). Paris, France: Société Française d'Ichtyologie, Muséum National d'Histoire Naturelle.

Karanth, K. U. (1995). Estimating tiger populations from camera -trap data using capture- recapture models. Biological Conservation, 71, 333-338.

Kenny, J. S. (1995). Views from the bridge: a memoir on the freshwater fishes of Trinidad., St. Joseph, Trinidad and Tobago: Julian S. Kenny.

Lasso, C. A., \& Lasso-Alcalá, O. M. (2011). Rivulus hartii. In C. A. Lasso \& P. Sánchez-Duarte (Eds.), Los peces del delta del Orinoco. Diversidad, bioecología, uso y conservación (pp. 274-275). Caracas, Venezuela: Fundación La Salle de Ciencias Naturales y Chevron C. A.

Lasso, C., Lew, D., Taphorn, D., Do Nascimiento, C., Lasso-Alcalá, O., Provenzano, F., \& Machado-Allison, A. (2004). Biodiversidad ictiológica continental de Venezuela. Parte I. Lista de especies y distribución por cuencas. Memoria de la Fundación La Salle de Ciencias Naturales, 159-160, 105-196.

Mago, F. (1970). Lista de los peces de Venezuela, incluyendo un estudio preliminar sobre la ictiogeografia del país. Caracas: Oficinal Nacional de Pesca, Ministerio de Agricultura y Cría.

Mesa-Zavala, E., Álvarez-Cárdenas, S., Galina-Tessaro, P., Troyo-Diéguez, E., \& Guerrero-Cárdenas, I. (2012). Vertebrados terrestres registrados mediante fototrampeo en arroyos estacionales y cañadas con agua superficial en un hábitat semiárido de Baja California
Sur, México. Revista Mexicana de Biodiversidad, 83(1), 235-245.

Nelson, J. S., Grande, T., \& Wilson, M. (2016). Fishes of the world. New Jersey, USA: John Wiley \& Sons.

Nirchio, M., Gómez, J., Oliveira, C., Ron, E., Nirchio, M. J., \& Villaroel, J. (2005). Rivulus hartii (Boulenger 1890) (Cyprinodontiformes: Rivulidae): especie de pez dulceacuícola amenazada en la isla de Margarita, Venezuela. Tecnociencia, 7(1), 135-142.

Parenti, L. R. (1981). A phylogenetic and biogeographic analysis of cyprinodontiform fishes (Teleostei, Atherinomorpha). Bulletin of the American Museum of Natural History, 168, 335-557.

Phillip, D. A. T., Taphorn, D. C., Holm, E., Gilliam, J. F., Lamphere, B. A., \& López-Fernández, H. (2013). Annotated list and key to the stream fishes of Trinidad \& Tobago. Zootaxa, 3711(1), 001-064.

Robins, C. R., Bailey, R. M., Bond, C. E., Brooker, J. R., Lachner, E. A., Lea, R. N., \& Scott, W. B. (1991). World fishes important to North Americans. Exclusive of species from the continental waters of the United States and Canada. American Fisheries Society Special Publication Series, 21, 243.

Rodríguez-Olarte, D., \& Taphorn, D. C. (2007). Los peces de Aroa y Yaracuy: una guía para su conservación. Recuperado de http://www.ucla.edu.ve/museopeces $/ 2 . \% 20$ Pagina $\% 20$ WEB $\% 20$ Peces $\% 20$ Aroa $\% 20$ Yaracuy\%202007/Rivulus.htm

Schultz, L. (1949). A further contribution to the ichthyology of Venezuela. Proceedings of the United States National Museum, 99, 1-211.

Seydack, A. H. W. (1984). Application of a photo-recording device in the census of larger rain forest mammals. South African Journal of Wildlife Research, $14,10-14$

Taphorn, D., Royero, R., Machado-Allison, A., \& MagoLeccia, F. (1997). Lista actualizada de los peces de agua dulce de Venezuela. In E. La Marca (Ed.), Vertebrados actuales y fósiles de Venezuela (pp. 55-100). Mérida, Venezuela: Serie Catálogo Zoológico de Venezuela.

Vermeulen, F. (2015). New World Killi Series Vol. 1: The killi's of the lost world. Portugal: Pastelaria Studios. 


\section{MATERIAL EXAMINADO}

MBUCV 12510: Estado Sucre, caño Yaguaina cerca de Guariquén (10.258200 -62.956531). Fecha de colecta 11-12-1980. Material examinado: 12 Hembras; 39 hembras juveniles; 17 intermedios y 2 machos.

MBUCV 20260: Estado Sucre, río Yacua, al O. de la Macuro. Fecha de colecta: 29-031990. Material examinado: 4 Hembras y 4 machos.

MBUCV 12445: Estado Sucre, Charca en el margen de la carretera a boca de Ajies, vecindario Mapuey, cerca de Boca de Ajies. Fecha de colecta: 07-12-1980. Material examinado: 5 Hembras; 11 hembras juveniles; 4 intermedios.

MBUCV 7001: Distrito Capital, quebrada Tribito (paratipos de Rivulus bondi Schultz 1949) población extinta. Fecha de colecta: 01-10-1938. Material examinado: 3 Hembras; 2 hembras juveniles; 4 intermedio y 1 macho.

MBUCV 24109: Estado Falcón, Pozo Azul, cerro de Chichiriviche, refugio de Cuare. Fecha de colecta 24-10-1992. Material examinado: 20 Hembras; 4 hembras juveniles; 3 intermedios y 8 machos.

MBUCV 35742: Estado Miranda, cordillera del interior, río Araira, sector la laguna, microcuenca del río Tuy (10.487948 /-66.462939). Fecha de colecta 19-042015. Material examinado: 3 Hembras; 2 hembras juveniles; 1 macho.

MCNG 17163: Estado Miranda, cañito en El Valle, debajo de la Urbanización Loma Larga, al sur de Caracas. (10.401524/66.829593). Fecha de colecta: 16-2-1987. Población extinta.

MBUCV 5005: Estado Miranda, vertiente costera, río Aricagua, quebrada Rebollero. Fecha de colecta 8-7-1967. Material examinado: 28 Hembras; 2 hembras juveniles; 3 intermedios y 12 machos.

MBUCV 35741: Estado Miranda, cordillera del interior, quebrada chuspita, carretera Guatire-Caucagua Miranda. Fecha de colecta: 22-12-1961. Material examinado:
7 Hembras; 2 hembras juveniles; 2 intermedio juvenil; 3 machos.

MBUCV 35738: Estado Yaracuy, Estación Biológica Hacienda la Guaquira, (10.17462 /-68.39208). Fecha de colecta 20-05-2010. Material examinado: 3 Hembras; 1 hembras juveniles; 2 intermedios.

MCNG 25655: Estado Yaracuy, caño $6 \mathrm{~km}$ al Norte de Marín en la vía a Crucito (Aroa). Fecha de colecta: 22-4-1991.

MCNG 25646: Estado Lara, quebrada el Oro, en el puente entre Licua y Aroa, vía Aroa. (10.267100/-69.039307). Fecha de colecta: 21-4-1991.

MBUCV 29496: Estado Nueva Esparta, represa San Juan Bautista (11.02985/ -63.938158) Fecha de colecta 29-10-2000. Material examinado: 2 Hembras; 1 intermedio y 4 machos.

MCNG 21345: Estado Nueva Esparta, vía a el Valle del Espíritu Santo, Cerro Copey, cerca de la capilla el valle, riachuelo el Valle. (10.985697/-63.888423) Fecha de colecta: 14-08-1989.

MCNG 8238: Isla Trinidad, cono en Palo Seco, en el pueblo. (10.099756/-61.619714) Fecha de colecta 22-06-1983. Material examinado: 2 Hembras; 2 hembras juveniles; 3 intermedios y 2 machos.

MBUCV 8886: Estado Sucre, río San Juan, San Fernando Distrito Montes. Fecha de colecta: 13-12-1992. Material examinado: 14 Hembras; 18 hembras juveniles; 12 intermedios.

MBUCV 8887: Estado Sucre, río San Juan, San Fernando Distrito Montes. Fecha de colecta: 13-12-1992. Material examinado: 16 Hembras; 3 intermedios y 7 machos.

MBUCV 20257: Estado Sucre, río Mapire, oeste de puente de Hierro, ensenada de Mapire. Fecha de colecta: 20-03-1990. Material examinado: 13 Hembras; 6 hembras juveniles; 4 intermedios.

MBUCV 20245: Estado Sucre, río los Mereyes, Afluentes del río Casanay, cerca de mundo nuevo. Fecha de colecta: 26-03-1990. Material examinado: 2 Hembras; 2 hembras juveniles; 2 intermedios y 2 machos. 